\title{
NOETHERIAN PI RINGS NOT MODULE-FINITE OVER ANY COMMUTATIVE SUBRING
}

\author{
J. J. SARRAILLE
}

\begin{abstract}
We construct a ring $R$ of $3 \times 3$ matrices over $k[x, y, z]$ which is prime, affine, Noetherian, and PI, but not finitely generated as a module nor integral over any commutative subring.
\end{abstract}

0. Introduction. How close do PI rings come to being commutative? Kaplansky's theorem, Posner's theorem, Artin's theorem, and the discovery of the existence of central polynomials are examples of deep results which have helped to answer that question (see [9, 7]). We also know that every semiprime PI ring can be embedded in $M_{n}(C)$ for some $n$ and for some commutative ring $C$, where $M_{n}(C)$ is the ring of $n \times n$ matrices with entries in $C$ (see [1]). On the other hand, there are affine PI rings which cannot be embedded in $M_{n}(C)$ for any $n$ or commutative ring $C$ (see $[2,10$, 13]). We know [8] that if the center of a semiprime PI ring $A$ is Noetherian then $A$ is finitely generated as a module over its center. However there are [6] prime, affine, Noetherian PI rings whose centers are not Noetherian, and they are not finitely generated as modules over their centers.

How close do Noetherian PI rings come to being commutative? When are they finitely generated as modules over some (not necessarily central) commutative subring? A few years ago George Bergman described a ring as follows: Let $k$ be a field with characteristic zero. Let $S=k\{u, v, w\}$ be the image of the free associative algebra in three indeterminates over $k$ obtained by taking the relations

$$
[u, v]=w,[u, w]=[v, w]=w^{2}=0 .
$$

Then $S$ is an affine, Noetherian PI ring which is not finitely generated as a module over any commutative subring - we will give a proof due to Bergman of this. Note that $S$ is not prime. In fact the ideal $P$ generated by $w$ is a nilpotent prime, and $S / P$ is isomorphic to the commutative polynomial ring $k[x, y]$.

In this paper we construct a ring $B$ which is isomorphic to $S$, and we use $B$ to construct a ring $R$ which is prime, affine, Noetherian, PI, but not finitely generated as a module over any commutative subring. Both $B$ and $R$ are described explicitly as rings of $3 \times 3$ matrices with entries in commutative polynomial rings. The existence of $R$ shows that prime Noetherian PI rings do not arise solely as finitely generated modules over commutative Noetherian subrings, which answers a question posed by L. Small.

Received by the editors April 27, 1981.

1980 Mathematics Subject Classification. Primary 16A12, 16A33, 16A38.

(C)1982 American Mathematical Society $0002-9947 / 81 / 0000-0347 / \$ 02.50$ 
1. Explication of the example. Let $k$ be a field with characteristic zero. Define three-by-three upper triangular matrices $X$ and $Y$ as follows:

$$
X=\left(\begin{array}{lll}
x & 1 & 0 \\
0 & x & 0 \\
0 & 0 & x
\end{array}\right), \quad Y=\left(\begin{array}{lll}
y & 0 & 0 \\
0 & y & 1 \\
0 & 0 & y
\end{array}\right),
$$

where $x$ and $y$ are commuting indeterminates. In order to verify that the example has the properties we claim it has, it will turn out to be helpful if we first prove the following proposition.

Proposition 1. Let $k\{X, Y\}=A$ be the algebra with 1 generated over $k$ by $X$ and Y. Define

$$
B=\left\{\left(\begin{array}{ccc}
f & f_{x} & h \\
0 & f & f_{y} \\
0 & 0 & f
\end{array}\right) \mid f, h \in k[x, y]\right\}
$$

where $k[x, y]$ is the polynomial ring and where $f_{x}$ and $f_{y}$ are respectively the partial derivatives $\partial f / \partial x$ and $\partial f / \partial y$. Then $A=B$.

Proof. Verify the following equalities:

$$
\begin{gathered}
X^{n}=\left(\begin{array}{ccc}
x^{n} & n x^{n-1} & 0 \\
0 & x^{n} & 0 \\
0 & 0 & x^{n}
\end{array}\right), \quad Y^{m}=\left(\begin{array}{ccc}
y^{m} & 0 & 0 \\
0 & y^{m} & m y^{m-1} \\
0 & 0 & y^{m}
\end{array}\right), \\
T=[X, Y]=\left(\begin{array}{ccc}
0 & 0 & 1 \\
0 & 0 & 0 \\
0 & 0 & 0
\end{array}\right), \quad[X, T]=[Y, T]=T^{2}=0 \\
X^{n} Y^{m}=\left(\begin{array}{ccc}
x^{n} y^{m} & n x^{n-1} y^{m} & m n x^{n-1} y^{m-1} \\
0 & x^{n} y^{m} & m x^{n} y^{m-1} \\
0 & 0 & x^{n} y^{m}
\end{array}\right)
\end{gathered}
$$

If we put $p=x^{n} y^{m}$, the last equality above says that

$$
X^{n} Y^{m}=\left(\begin{array}{ccc}
p & p_{x} & p_{x y} \\
0 & p & p_{y} \\
0 & 0 & p
\end{array}\right)
$$

where $p_{x y}$ is the mixed second degree partial derivative $\partial^{2} p / \partial x \partial y$. By an obvious linearity argument, if $f \in k[x, y]$, say $f=\sum \alpha_{n m} x^{n} y^{m}$ where each $\alpha_{n m} \in k$, then

$$
F=\sum \alpha_{n m} X^{n} Y^{m}=\left(\begin{array}{ccc}
f & f_{x} & f_{x y} \\
0 & f & f_{y} \\
0 & 0 & f
\end{array}\right)
$$

Also, if $d=\Sigma \beta_{i j} x^{i} y^{j}$, then

$$
D T=\left(\sum \beta_{i j} X^{i} Y^{j}\right)(X Y-Y X)=\left(\begin{array}{ccc}
d & d_{x} & d_{x y} \\
0 & d & d_{y} \\
0 & 0 & d
\end{array}\right)\left(\begin{array}{lll}
0 & 0 & 1 \\
0 & 0 & 0 \\
0 & 0 & 0
\end{array}\right)=\left(\begin{array}{lll}
0 & 0 & d \\
0 & 0 & 0 \\
0 & 0 & 0
\end{array}\right) \text {. }
$$


Thus if we choose $d=h-f_{x y}$, the arbitrary element

$$
\left(\begin{array}{ccc}
f & f_{x} & h \\
0 & f & f_{y} \\
0 & 0 & f
\end{array}\right)
$$

is equal to $F+D T$. Since $F, D$, and $T$ are elements of $A=k\{X, Y\}$, we have proved that $B \subset A$.

To prove $A \subset B$, note that an element $K \in A$ has the form

$$
K=\sum \lambda_{n m} X^{n} Y^{m}+\Gamma
$$

where $\Gamma$ is an element of the commutator ideal. Since $[X, Y]=T$ is a central element of $A$, the commutator ideal is just $A T$ and so $\Gamma=G T$ where $G$ is some element of $A$. Since $X$ and $Y$ are upper triangular, so is $G$. Thus we have

$$
K=\left(\begin{array}{ccc}
b & b_{x} & b_{x y} \\
0 & b & b_{y} \\
0 & 0 & b
\end{array}\right)+\left(\begin{array}{ccc}
g_{11} & g_{12} & g_{13} \\
0 & g_{22} & g_{23} \\
0 & 0 & g_{33}
\end{array}\right)\left(\begin{array}{ccc}
0 & 0 & 1 \\
0 & 0 & 0 \\
0 & 0 & 0
\end{array}\right)=\left(\begin{array}{ccc}
b & b_{x} & b_{x y}+g_{11} \\
0 & b & b_{y} \\
0 & 0 & b
\end{array}\right)
$$

where $b=\Sigma \lambda_{n m} x^{n} y^{m}$ and $G$ is the matrix $\left(\left(g_{i j}\right)\right)$. Thus $A \subset B$.

We claim $S \cong B$. To see this, note that because of the relations $(0)$, we can write any element $s \in S$ in the form

$$
s=\sum \alpha_{i j} u^{i} v^{j}+s_{1} w
$$

where each $\alpha_{i j} \in k$ and $s_{1} \in S$. Naturally we can write $s_{1}$ in the same form:

$$
s_{1}=\sum \beta_{i j} u^{i} v^{j}+s_{2} w
$$

and if we multiply both sides of (6) by $w$, we get

$$
s_{1} w=\left(\sum \beta_{i j} u^{i} v^{j}\right) w .
$$

Thus using (5) and (7), we see that any $s \in S$ can be written:

$$
s=\sum \alpha_{i j} u^{i} v^{j}+\left(\sum \beta_{i j} u^{i} v^{j}\right) w .
$$

Another obvious fact is that there is a ring homomorphism $\pi: S \rightarrow B$ given by $\pi(u)=X$ and $\pi(v)=Y$. This follows trivially from (2), and from the fact that (0) gives the defining relations of $S$. Our claim is that $\pi$ is an isomorphism. If $s \in \operatorname{Ker}(\pi)$, then using (8) we get

$$
\begin{aligned}
0= & \pi(s)=\sum \alpha_{i j} X^{i} Y^{j}+\left(\sum \beta_{i j} X^{i} Y^{j}\right) T=\left(\begin{array}{ccc}
f & f_{x} & f_{x y} \\
0 & f & f_{y} \\
0 & 0 & f
\end{array}\right) \\
& +\left(\begin{array}{ccc}
g & g_{x} & g_{x y} \\
0 & g & g_{y} \\
0 & 0 & g
\end{array}\right)\left(\begin{array}{lll}
0 & 0 & 1 \\
0 & 0 & 0 \\
0 & 0 & 0
\end{array}\right)=\left(\begin{array}{ccc}
f & f_{x} & f_{x y}+g \\
0 & f & f_{y} \\
0 & 0 & f
\end{array}\right)
\end{aligned}
$$

where $f=\Sigma \alpha_{i j} x^{i} y^{j}$ and $g=\Sigma \beta_{i j} x^{i} y^{j}$. So $\pi(s)=0$ clearly implies $f=0$ and $g=0$. Thus all of the $\alpha_{i j}$ and $\beta_{i j}$ are zero, which by (8) implies $s=0$. 
By (2), $T$ is a central nilpotent element of $B$. It is obvious that

$$
B T=\left\{\left(\begin{array}{lll}
0 & 0 & g \\
0 & 0 & 0 \\
0 & 0 & 0
\end{array}\right) \mid g \in k[x, y]\right\}
$$

and clearly $B / B T \cong k[x, y]$, for the mapping from $B$ to $k[x, y]$ given by $X \rightarrow x$ and $Y \rightarrow y$ has kernel $B T$. This implies that $B T$ is the nil radical of $B$, and that $B$ is Noetherian.

In order to prove that $B$ is not finitely generated over any commutative subring, we need to look closely at what it means for two elements of $B$ to commute. So suppose

$$
F=\left(\begin{array}{ccc}
f & f_{x} & \varphi \\
0 & f & f_{y} \\
0 & 0 & f
\end{array}\right) \quad \text { and } \quad G=\left(\begin{array}{ccc}
g & g_{x} & \psi \\
0 & g & g_{y} \\
0 & 0 & g
\end{array}\right)
$$

are arbitrary elements of $B$. An easy computation shows that

$$
[F, G]=\left(\begin{array}{ccc}
0 & 0 & f_{x} g_{y}-f_{y} g_{x} \\
0 & 0 & 0 \\
0 & 0 & 0
\end{array}\right)=\left(f_{x} g_{y}-f_{y} g_{x}\right) T
$$

If $B$ were finitely generated as a module over some commutative subring $C$, then $\bar{B}=B / B T \cong k[x, y]$ would be a finitely generated $\bar{C}$-module where $\bar{C}$ is the image of $C$ in $\bar{B}$. By the Artin-Tate lemma (see [3]), $\bar{C}$ would be a finitely generated $k$-algebra. The Noether normalization lemma would imply that $\bar{C}$ is finitely generated as a module over a subalgebra of the form $k[f, g]$ where $f$ and $g$ are algebraically independent elements of $B / B T$.

Now assuming $F, G$ are elements of $C$ such that $\bar{F}=f$ and $\bar{G}=g$, we would get $0=[F, G]=\left(f_{x} g_{y}-f_{y} g_{x}\right) T$; so $0=f_{x} g_{y}-f_{y} g_{x}$ i.e.

$$
0=\frac{\partial f}{\partial x} \frac{\partial g}{\partial y}-\frac{\partial f}{\partial y} \frac{\partial g}{\partial x}=\delta(g)
$$

where $\delta$ is the $k$-derivation of $k[x, y]$ defined by

$$
\delta(p)=\frac{\partial f}{\partial x} \frac{\partial p}{\partial y}-\frac{\partial f}{\partial y} \frac{\partial p}{\partial x}, \quad p \in k[x, y] .
$$

Obviously $\delta(f)=0$; so $\delta$ vanishes on all of $k[f, g]$. But since $k[x, y]$ is integral over $k[f, g], \delta$ must also vanish on $k[x, y]$ : say $s \in k[x, y]$ and $0=q(s)$ where $q$ is some monic polynomial with coefficients in $k[f, g]$. Then

$$
0=\delta(0)=\delta(q(s))=q^{\prime}(s) \delta(s) .
$$

Since $\operatorname{char}(k)=0$, we can assume that $q^{\prime}(s) \neq 0$ and that therefore $\delta(s)=0$; so $\delta$ vanishes on all elements of $s \in k[x, y]$. But $\delta(y)=\partial f / \partial x$ and $\delta(x)=-\partial f / \partial y$, implying $f \in k$, which contradicts the fact that $\bar{B}=k[x, y]$ is integral over $k[f, g]$. Thus $B$ is not finitely generated as a module over any commutative subring.

Note also that if $B$ were integral over some commutative subring $C$, then $\bar{B}$ would be integral and hence finitely generated as a module over $\bar{C}$. Thus the argument above also proves $B$ is not integral over any commutative subring. 
Now put $D=k[z]\{X, Y\}$ where $z$ is an indeterminate that commutes with $x$ and $y$. Since $D / D z \cong B$, it is clear that $D$ is also not finitely generated as a module over any commutative subring. Since $D \cong B[z], D$ is Noetherian by the Hilbert basis theorem. Let $I$ be the ideal of the ring $(k[x, y, z])_{3}$ of three-by-three matrices over $k[x, y, z]$ defined by

$$
I=\left\{\left(\begin{array}{lll}
z h_{11} & z h_{12} & z h_{13} \\
z h_{21} & z h_{22} & z h_{23} \\
z h_{31} & z h_{32} & z h_{33}
\end{array}\right) \mid h_{i j} \in k[x, y, z]\right\} .
$$

Now $I$ has the same (classical) ring of quotients as does $(k[x, y, z])_{3}$, namely $(k(x, y, z))_{3}$-the ring of three-by-three matrices over the quotient field of $k[x, y, z]$. Therefore, Goldie's theorem implies that the ring $R=D+I$ is a prime PI ring. $I$ is an ideal of $R$, and $R / I$ is isomorphic to $B$; so $R$ is not finitely generated as a module over any commutative subring, nor is $R$ integral over any commutative subring. Now note that we have the result

Proposition 2. If $D=k[z]\{X, Y\}$, then

$$
D=\left\{\left(\begin{array}{ccc}
f & f_{x} & g \\
0 & f & f_{y} \\
0 & 0 & f
\end{array}\right) \mid f, g \in k[x, y, z]\right\} .
$$

And Proposition 2 is proved just as Proposition 1 is proved. Verify the following relations:

$$
\begin{aligned}
& \left(\begin{array}{ccc}
z f & 0 & 0 \\
0 & 0 & 0 \\
0 & 0 & 0
\end{array}\right)=\left(\begin{array}{ccc}
f & f_{x} & 0 \\
0 & f & f_{y} \\
0 & 0 & f
\end{array}\right)\left(\begin{array}{ccc}
z & 0 & 0 \\
0 & 0 & 0 \\
0 & 0 & 0
\end{array}\right) \quad \forall f \in k[x, y, z] \\
& \left(\begin{array}{ccc}
0 & 0 & 0 \\
z g & 0 & 0 \\
0 & 0 & 0
\end{array}\right)=\left(\begin{array}{ccc}
g & g_{x} & 0 \\
0 & g & g_{y} \\
0 & 0 & g
\end{array}\right)\left(\begin{array}{ccc}
0 & 0 & 0 \\
z & 0 & 0 \\
0 & 0 & 0
\end{array}\right)-\left(\begin{array}{ccc}
z g_{x} & 0 & 0 \\
0 & 0 & 0 \\
0 & 0 & 0
\end{array}\right) \\
& \forall g \in k[x, y, z], \\
& \left(\begin{array}{ccc}
0 & 0 & 0 \\
0 & 0 & 0 \\
z h & 0 & 0
\end{array}\right)=\left(\begin{array}{ccc}
h & h_{x} & 0 \\
0 & h & h_{y} \\
0 & 0 & h
\end{array}\right)\left(\begin{array}{lll}
0 & 0 & 0 \\
0 & 0 & 0 \\
z & 0 & 0
\end{array}\right)-\left(\begin{array}{ccc}
0 & 0 & 0 \\
z h_{y} & 0 & 0 \\
0 & 0 & 0
\end{array}\right) \\
& \forall h \in k[x, y, z] \text {. }
\end{aligned}
$$

Taken together, (9), (10), and (11) imply that all elements of $I$ of the form

$$
\left(\begin{array}{lll}
z f & 0 & 0 \\
z g & 0 & 0 \\
z h & 0 & 0
\end{array}\right)
$$


are contained in the finitely generated left $D$-module

$$
D\left(\begin{array}{lll}
z & 0 & 0 \\
0 & 0 & 0 \\
0 & 0 & 0
\end{array}\right)+D\left(\begin{array}{lll}
0 & 0 & 0 \\
z & 0 & 0 \\
0 & 0 & 0
\end{array}\right)+D\left(\begin{array}{lll}
0 & 0 & 0 \\
0 & 0 & 0 \\
z & 0 & 0
\end{array}\right) .
$$

By extending the calculations above in an obvious way, one can prove that $I$ is a finitely generated left $D$-module. So $R=D+I$ is also a finitely generated left $D$-module. That proves $R$ is Noetherian and affine, and we have now completed the proof of

THEOREM. There exists a prime, affine, Noetherian PI ring with Krull dimension three which is not integral nor finitely generated as a module over any commutative subring.

By [5], three is the lowest Krull dimension possible for such an example. Even if the ring is allowed to be "affine" over an arbitrary commutative ring, an example like $R$ with Krull dimension one cannot exist [12]. The reader may find it interesting to note that $B$ and $R$ and the example of $\$ 3$ of [6] are all very similar to each other.

2. Open problems. The problem of completely characterizing Noetherian PI rings remains open. We would like to know if there is an affine, Noetherian PI ring which cannot be embedded in $M_{n}(C)$ for any $n$ nor any commutative ring $C$ (see [4 and 11]).

We would also like to know if there is a ring which is prime, PI, Noetherian, and affine, but not finitely generated as a module over any commutative Noetherian ring. Oddly enough, the ring $R$ which we have constructed is a finitely generated module over the commutative polynomial ring $k[x, y, z]$. One defines $x \cdot F=X F$ and $y \cdot F=F Y$ for elements $F \in R$. This module action does not preserve the one-sided ideals of $R$, and the mapping $p \rightarrow p \cdot 1$ from $k[x, y, z]$ to $R$ is not a ring homomorphism. However, the module action does preserve the two-sided ideals of $R$. It is possible to prove that any prime PI ring $A$ which is finitely generated as a module over a commutative Noetherian ring is right and left Noetherian if the module action preserves the two-sided ideals of $A$ (we omit the proof, but it is an easy consequence of Proposition 1.1 of [6]). We can therefore view our ring $R$ as being Noetherian by virtue of the fact that it is module-finite over $k[x, y, z]$. We wonder if prime Noetherian PI rings can arise in any other way.

ACKNOWLEDGEMENTS. The author thanks G. Bergman for his excellent example. Thanks also to L. Small for suggesting the topic of this paper, and to both L. Small and A. Wadsworth for their many comments and helpful suggestions.

\section{REFERENCES}

1. S. A. Amitsur, An imbedding of PI rings, Proc. Amer. Math. Soc. 3 (1952), 3-9.

2. A noncommutative Hilbert basis theorem and subrings of matrices, Trans. Amer. Math. Soc. 149 (1970), 133-142.

3. E. Artin and J. T. Tate, $A$ note on finite ring extensions, J. Math. Soc. Japan 3 (1951), 74-77.

4. G. Bergman, Some examples in PI ring theory, Israel J. Math. 18 (1974), 257-277.

5. A. Braun, A note on Noetherian p.i. rings, Proc. Amer. Math. Soc. 83 (1981), 670-672.

6. G. Cauchon, Anneaux semi-premiers, Noethériens, à identités polynomiâles, Bull. Soc. Math. France 104 (1976), 99-111. 
7. P. M. Cohn, Algebra 2, Wiley, London, 1977.

8. E. Formanek, Noetherian PI rings, Comm. Algebra 1 (1974), 79-86.

9. I. Kaplansky, "Problems in the theory of rings", revisited, Amer. Math. Monthly 77 (1970), 445-454.

10. J. Lewin, On some infinitely presented associative algebras and an example of L. Small, J. Austral. Math. Soc. 16 (1973), 290-293.

11. __ A matrix representation for associative algebras. I, Trans. Amer. Math. Soc. 188 (1974), 293-308.

12. J. J. Sarraille, Module-finiteness of low dimensional PI rings, Pacific J. Math. (to appear).

13. L. Small, An example in PI rings, J. Algebra 17 (1971), 434-436.

Department of Mathematics, University of TeXas, Austin, TeXas 78712 